\title{
The Real Impact of Bridging or Downstaging on Survival Outcomes after Liver Transplantation for Hepatocellular Carcinoma
}

\author{
Sunyoung Lee ${ }^{a} \quad K^{\prime} y o u n g$ Won Kim ${ }^{b}$ Gi-Won Song ${ }^{c}$ Jae Hyun Kwon ${ }^{c}$ \\ Shin Hwang ${ }^{c}$ Ki-Hun Kim ${ }^{c}$ Chul-Soo Ahn ${ }^{c}$ Deok-Bog Moon ${ }^{c}$ \\ Gil-Chun Park ${ }^{c}$ Sung-Gyu Lee ${ }^{c}$ \\ aDepartment of Radiology and Research Institute of Radiological Science, Severance \\ Hospital, Yonsei University College of Medicine, Seoul, Republic of Korea; ${ }^{b}$ Department of \\ Radiology and Research Institute of Radiology, Asan Medical Center, University of Ulsan \\ College of Medicine, Seoul, Republic of Korea; 'Department of Surgery, Asan Medical \\ Center, University of Ulsan College of Medicine, Seoul, Republic of Korea
}

\section{Keywords}

Liver cancer $\cdot$ Transplantation $\cdot$ Recurrence $\cdot$ Survival $\cdot$ mRECIST

\begin{abstract}
Introduction: There is no consensus regarding selection criteria on liver transplantation (LT) for hepatocellular carcinoma (HCC), especially for living donor liver transplantation, although emerging evidence has been found for the effectiveness of bridging or downstaging. Objective: We evaluated the long-term outcomes of patients who underwent LT with or without bridging or downstaging for HCC. Methods: This retrospective study included 896 LT recipients with HCC between June 2005 and May 2015. Recurrence-free survival (RFS), overall survival (OS), and their associated factors were evaluated. Results: The 5-year RFS in the full cohort of 896 patients was $82.4 \%$, and the OS was $85.3 \%$. In patients with initial Organ Procurement and Transplantation Network (OPTN) T1 and T2, the 5-year RFS and OS did not significantly differ between LT groups with and without bridging (all $p \geq 0.05$ ). The 5 -year RFS and OS of OPTN T3 patients with successful downstaging were not significantly different from those of patients with OPTN T2 with primary LT ( $p=0.070$ and $p=0.185)$, but were significantly higher than in patients with OPTN T3 with downstaging failure and initial OPTN T1 or T2 with progression (all $p<0.001$ ). In the multivariate analysis, last alpha-fetoprotein before $\mathrm{LT} \geq 70 \mathrm{ng} / \mathrm{mL}$ (hazard ratio [HR]: 1.77, $p=0.001 ; \mathrm{HR}: 1.72, p=0.004$ ), pretransplant HCC status exceeding the Milan criteria (HR: $5.12, p<0.001$; HR: 3.31, $p<0.001$ ), and positron emission
\end{abstract}

Gi-Won Song and Kyoung Won Kim have contributed equally to this work. 


\begin{tabular}{ll|l}
\cline { 2 - 2 } Liver Cancer 2020;9:721-733 \\
\cline { 2 - 3 } DOI: 10.1159/000507887 & $\begin{array}{l}\text { @ } 2020 \text { The Author(s). Published by S. Karger AG, Basel } \\
\text { www.karger.com/lic }\end{array}$ \\
\hline
\end{tabular}

Lee et al.: Bridging or Downstaging and LT for HCC

tomography positivity (HR: 2.57, $p<0.001$; HR: 2.57, $p<0.001$ ) were independent predictors for worse RFS and OS. Conclusions: The impact of bridging therapy on survival outcomes is limited in patients with early-stage HCC, whereas OPTN T1 or T2 with progression provides worse prognosis. OPTN T3 should undergo LT after successful downstaging, and OPTN T3 with successful downstaging allows for acceptable long-term posttransplant outcomes.

(C) 2020 The Author(s)

Published by S. Karger AG, Basel

\section{Introduction}

Liver transplantation (LT) is an established therapy for patients with early-stage hepatocellular carcinoma (HCC) and cirrhosis. LT is, theoretically, the optimal treatment as it provides a chance to cure not only the HCC but also the underlying liver disease simultaneously. However, limited availability of liver grafts and tumor recurrence are critical limiting factors for performing LT in HCC patients. Accordingly, strict eligibility guidelines for deceased donor liver transplantation (DDLT), such as the Milan criteria, have been developed to reduce the posttransplant HCC recurrence [1]. The introduction of the Milan criteria for the national allocation system markedly decreased HCC recurrence but increased both dropout rate on the waiting list and the waitlist mortality for DDLT. In contrast to DDLT, liver grafts used in living donor liver transplantation (LDLT) are not considered a public resource, and therefore the selection criteria for LDLT in patients with HCC may be expanded beyond the Milan criteria while balancing recipient survival benefit and donor risk [2]. However, an international consensus on how far to expand selection criteria for LDLT donors has not been reached [3].

When describing neoadjuvant treatments for HCC in patients on the LT waitlist, "bridging" is defined as treatment to control tumor growth in patients within accepted transplant criteria during the waitlist period, while "downstaging" refers to treatment to reduce tumor burden to meet acceptable transplant criteria [4]. Locoregional therapy (LRT) has been implemented in bridging therapy to minimize the risk of HCC progression on the waiting list, while downstaging is to reduce the tumor size and number in patients initially exceeding the Milan criteria in order to be considered for LT [3-5]. Patients with intermediate/advanced-stage HCC might become reasonable candidates for LT after successful downstaging (SD) with LRT and then achieve comparable survival outcomes to patients who met the Milan criteria without downstaging $[3,4]$.

The objective radiologic response evaluation is essential to assess treatment effectiveness and subsequent patient outcomes. The main objective of effective LRT is to induce necrosis of a viable tumor regardless of shrinkage of the entire tumor. Therefore, new methods including the concept of viable tumor (enhancing portion in the arterial phase) have been proposed, and the modified Response Evaluation Criteria in Solid Tumors (mRECIST) criteria have been the most common criteria to evaluate radiologic responses of HCC treated with LRT [4-6].

There have been emerging evidence on the effectiveness of LRT as a bridging or downstaging strategy from the prior reports including the recent meta-analyses [7-9]. However, a majority of previous studies are limited by the small study population, short follow-up duration, and noncomparative design. The definition of SD as well as the methods to assess radiologic response also varied among the previous studies [5, 8]. Furthermore, there are few comparative outcome data for patients with Organ Procurement and Transplantation Network (OPTN) T1 HCC with LRT versus observation and for patients with initial OPTN T3 HCC with and without downstaging in terms of post-LT recurrence or survival. Considering these factors, we evaluated the long-term outcomes of a large number of patients who underwent LT with or without bridging or downstaging for HCC by stratifying according to initial OPTN stage and status after LRT using mRECIST.

\section{Karger ${ }^{\prime \prime}$}




\begin{tabular}{l|l}
\hline Liver Cancer 2020;9:721-733 \\
\hline DOI: 10.1159/000507887 & $\begin{array}{l}\text { @ 2020 The Author(s). Published by S. Karger AG, Basel } \\
\text { www.karger.com/lic }\end{array}$ \\
\hline
\end{tabular}

Lee et al.: Bridging or Downstaging and LT for HCC

\section{Materials and Methods}

Patients

From a prospectively collected database of LT recipients, we retrieved 1,016 patients who underwent LT for HCC without extrahepatic metastasis between June 2005 and May 2015. The inclusion criteria of this study were as follows: (1) adult patients (age $\geq 18$ years), (2) patients who underwent contrast-enhanced multiphasic computed tomography (CT) or magnetic resonance imaging (MRI) at the time of initial HCC diagnosis and within 3 months prior to LT, and (3) patients who had available serum alpha-fetoprotein (AFP) levels measured within 1 month prior to LT. The exclusion criteria were as follows: (1) pediatric LT recipients, (2) patients who had macrovascular invasion detected in imaging, and (3) patients who were diagnosed with combined HCC-cholangiocarcinoma or cholangiocarcinoma on pathology after LT. Finally, 896 patients consisting of 829 LDLT recipients and 67 DDLT recipients were included in this study.

Image Analysis

Board-certified abdominal radiologists retrospectively reviewed baseline and follow-up imaging. The initial diagnosis of HCC was based on multiphasic contrast-enhanced CT or MRI showing typical hallmarks (>1 cm with arterial hypervascularity and portal venous or delayed phase washout) $[4,10,11]$. Tumor measurement after LRT was performed using the mRECIST criteria, which is the longest axial diameter of the viable portion (enhancement in the arterial phase), not including the area of necrosis [12] (see online suppl. Fig. 1; for all online suppl. material, see www.karger.com/doi/10.1159/000507887). Radiologic complete response after LRT was defined as disappearance of any intratumoral arterial enhancement [12]. Positron emission tomography (PET) scans were visually assessed whether the ${ }^{18} \mathrm{~F}$-fluorodeoxyglucose uptake in tumor was (PET positivity) or was not (PET negativity) significantly higher than in the surrounding noncancerous hepatic tissue [13].

\section{Subgroups according to OPTN Stage and LRT}

Patients were stratified according to initial OPTN stage based on imaging and LT with or without LRT. OPTN T1 was defined as a single HCC between 1 and $2 \mathrm{~cm}$. OPTN T2 was defined as either a single HCC between 2 and $5 \mathrm{~cm}$ or 2 or 3 HCCs each between 1 and $3 \mathrm{~cm}$ and considered the Milan criteria. OPTN T3 stage was equivalent to beyond the Milan criteria. SD was defined as a reduction in the number and size of viable tumors to within the Milan criteria. Downstaging failure (DF) was defined as when the number and size of viable tumors continued to exceed the Milan criteria after LRT. Progression was defined by an increase in the number or size of viable tumors from within the Milan criteria to beyond the Milan criteria despite LRT.

\section{Pathologic Analysis}

Data on histologic grade, microvascular invasion (MVI), and satellite nodule were extracted from pathologic reports of the explanted livers. Histologic grade was classified according to the Edmondson-Steiner grading system. MVI was defined as a tumor within a vascular space lined by the endothelium that was visible only with microscopy. Satellite nodule was defined as a microscopic nodule of HCC separated from the tumor by an interval (within $2 \mathrm{~cm}$ ) of uninvolved liver parenchyma.

\section{Transplantation Procedure and Follow-Up}

Specific surgical techniques and postoperative management including immunosuppression protocols for LT recipients have been published previously [14]. Mammalian target of rapamycin inhibitor as immunosuppressive agents was not used during the study period. Detailed follow-up protocols based on the risk of HCC recurrence have been described in previous reports [15]. Routine follow-up monitoring included regular radiologic (dynamic liver CT and chest radiography) and serologic analyses (AFP and protein induced by vitamin $\mathrm{K}$ absence or antagonist-II levels). MRI was performed when follow-up CT images suggested recurrence. If extrahepatic recurrence was suspected based on clinical symptoms or an unexplained elevation of a tumor marker level, the patients underwent chest CT, whole-body bone scintigraphy, and PET.

\section{Ethical Consideration}

Each transplantation procedure was evaluated and approved by the local authorities and the Korean Network for Organ Sharing affiliated with the Ministry of Health and Welfare of the Republic of Korea, and this study was reviewed and approved by the institutional review board of our institution. The study protocol

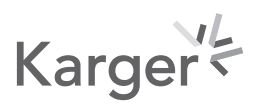




\section{Liver Cancer}

Table 1. Characteristics of the study population $(n=896)$

\begin{tabular}{|c|c|}
\hline \multicolumn{2}{|c|}{ Liver Cancer 2020;9:721-733 } \\
\hline DOI: 10.1159/000507887 & $\begin{array}{l}\text { (c) } 2020 \text { The Author(s). Published by S. Karger AG, Basel } \\
\text { www.karger.com/lic }\end{array}$ \\
\hline
\end{tabular}

Lee et al.: Bridging or Downstaging and LT for HCC

\begin{tabular}{|c|c|}
\hline Patients & \\
\hline Age, years ${ }^{\mathrm{a}}$ & $53.8 \pm 6.3$ \\
\hline Male & $760(84.8)$ \\
\hline Etiology of liver disease & \\
\hline Hepatitis B & $786(87.7)$ \\
\hline Hepatitis C & $49(5.5)$ \\
\hline Alcohol & $38(4.2)$ \\
\hline Others & $23(2.6)$ \\
\hline Child-Pugh classification & \\
\hline Class A & $354(39.5)$ \\
\hline Class B & $339(37.8)$ \\
\hline Class C & $203(22.7)$ \\
\hline MELD score $^{\mathrm{b}}$ & $11(8-16)$ \\
\hline Last AFP before $\mathrm{LT}, \mathrm{ng} / \mathrm{mL}^{\mathrm{b}}$ & $10.9(4.4-53.7)$ \\
\hline Tumor characteristics on pretransplant imaging & \\
\hline Diameter of the largest viable tumor ${ }^{\mathrm{a}}$ & $1.6 \pm 1.6$ \\
\hline Viable tumor, ${ }^{a} n$ & $1.4 \pm 2.6$ \\
\hline PET -2 & $n=781$ \\
\hline Positivity & $216(27.7)$ \\
\hline Types of LT & \\
\hline DDLT & $67(7.5)$ \\
\hline LDLT & $829(92.5)$ \\
\hline Primary LT (treatment-naïve HCC) & $208(23.2)$ \\
\hline LT after treatment & $n=688$ \\
\hline Types of treatment & \\
\hline cTACE alone & $409(59.4)$ \\
\hline RFA alone & $50(7.3)$ \\
\hline Resection alone & $13(1.9)$ \\
\hline Radiation alone & $5(0.7)$ \\
\hline Combination & $211(30.7)$ \\
\hline Radiologic complete response after treatment & $323(46.9)$ \\
\hline Tumor characteristics on pathology & $n=706$ \\
\hline Edmondson grade & \\
\hline I or II & $293(41.5)$ \\
\hline III or IV & $413(58.5)$ \\
\hline MVI & $111(15.7)$ \\
\hline Satellite nodule & $67(9.5)$ \\
\hline Interval from initial HCC diagnosis to LT, months ${ }^{b}$ & $9(3-28)$ \\
\hline
\end{tabular}

Unless otherwise indicated, data represent number of patients with percentage in parentheses. MELD, Model for End-Stage Liver Disease; AFP, alpha-fetoprotein; LT, liver transplantation; PET, positron emission tomography; DDLT, deceased donor liver transplantation; LDLT, living donor liver transplantation; HCC, hepatocellular carcinoma; cTACE, conventional transarterial chemoembolization; RFA, radiofre-

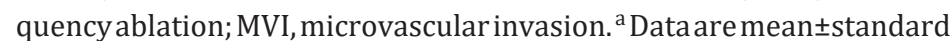
deviation. ${ }^{\mathrm{b}}$ Data are median with interquartile ranges in parentheses.

conforms to the ethical guidelines of the 1975 Declaration of Helsinki as reflected in a priori approval by the institution's human research committee. The requirement for informed consent was waived owing to the retrospective nature of the study.

\section{Statistical Analysis}

Categorical variables were compared by using the $\chi^{2}$ test or Fisher's exact test. Continuous data were evaluated using the 2-sample $t$ test or Mann-Whitney U test. Recurrence-free survival (RFS) was measured from the date of LT until HCC recurrence (intra- or extrahepatic recurrence) or the final documented date of 
no evidence of tumor recurrence by imaging. Overall survival (OS) was defined as the interval between LT and death or the date of the last follow-up visit. RFS and OS were estimated using Kaplan-Meier methods and were compared using the log-rank test. Univariate and multivariate analyses of RFS and OS were evaluated by using a Cox proportional hazards model. Variables with a $p$ value of $<0.05$ in univariate analysis were included in the multivariate analysis. Statistical analyses were performed using SPSS version 23.0 (IBM, Armonk, NY, USA) and MedCalc version 16.2.1 (MedCalc Software, Ostend, Belgium). A $p$ value of $<0.05$ was considered statistically significant.

\section{Results}

\section{Characteristics of the Study Population}

Demographic and clinical characteristics of 896 patients are summarized in Table 1. There were 760 males (mean age: 53.6 years; range: $31-69$ ) and 136 females (mean age: 54.7 years; range: 35-71), with an overall mean age of 53.8 years (range: 31-71). The median Model for End-Stage Liver Disease score was 11 (interquartile range [IQR]: 8-16). Of these patients, $208(23.2 \%)$ patients underwent primary LT for treatment-naïve HCC, and the remaining $688(76.8 \%)$ patients underwent LT after LRT. The most commonly used LRT was transarterial chemoembolization alone (59.4\%), followed by combination therapy (30.7\%) and radiofrequency ablation alone (7.3\%). The median interval from initial HCC diagnosis to LT was 9 months (IQR: 3-28).

Recurrence-Free Survival and Overall Survival

During the follow-up period (median: 89 months; IQR: 58-118), HCC recurrence after LT developed in $17.7 \%$ (159 of 896) of patients. Early recurrence $(<1$ year) occurred in 96 $(60.4 \%)$ patients, and late recurrence ( $>1$ year) occurred in $63(39.6 \%)$ patients. Forty-eight $(30.2 \%)$ patients had intrahepatic recurrence, and 111 (69.8\%) had extrahepatic recurrence. The 1-, 3-, 5-, and 10-year RFS rates in the full cohort of 896 transplanted HCC patients were 89.8, 84.7, 82.4, and 79.7\%, respectively. A total of 149 (16.6\%) patients died during the follow-up period, and 110 experienced HCC-related death. The 1-, 3-, 5-, and 10-year OS rates in the full cohort of 896 transplanted HCC patients were 96.2, 87.7, 85.3, and 82.3\%, respectively.

\section{Bridging or Downstaging and Primary LT on RFS and OS}

When patients were stratified according to initial OPTN stage based on imaging and LT with or without bridging or downstaging for HCC, 91 patients were initial OPTN T1 HCC with bridging therapy, 54 patients were initial OPTN T1 HCC with primary LT, 350 patients were initial OPTN T2 HCC with bridging therapy, 123 patients were initial OPTN T2 HCC with primary LT, 144 patients were initial OPTN T3 HCC with SD, 31 patients were initial OPTN T3 HCC with primary LT, 69 patients were initial OPTN T3 HCC with DF, and 34 patients were initial OPTN T1 or T2 HCC with progression (online suppl. Fig. 2). The RFS and OS rates of patients who underwent LT with or without bridging or downstaging for HCC by stratifying according to initial OPTN stage and status after LRT are presented in Figures 1 and 2. In patients with initial OPTN T1 and T2, the 5-year RFS and OS rates did not show statistically significant difference between LT with bridging therapy and primary LT (OPTN T1: 94.0 vs. $100 \%, p=0.050$, and 89.9 vs. $94.2 \%, p=0.247$; OPTN T2: 90.7 vs. $90.5 \%, p=0.954$, and 92.0 vs. $91.9 \%, p=0.411$ ). The 1-, 3-, 5-, and 10-year RFS and OS rates of patients with initial OPTN T3 with SD (88.1, 85.2, 83.5, and 78.9; 94.4, 84.0, 83.3, and 81.6\%, respectively) were not significantly different from those of patients with initial OPTN T2 with primary LT $(92.6,91.7$, 90.5 , and 87.6; 95.1, 91.9, 91.9, and 87.7\%, respectively) ( $p=0.070$ and $p=0.185$ ). In patients

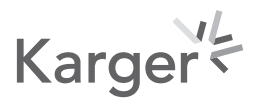




\begin{tabular}{ll|l} 
& \multicolumn{3}{l}{ Liver Cancer 2020;9:721-733 } \\
\cline { 2 - 3 } LOI: 10.1159/000507887 & $\begin{array}{l}\text { ( ) 2020 The Author(s). Published by S. Karger AG, Basel } \\
\text { www.karger.com/lic }\end{array}$ \\
\hline
\end{tabular}

Lee et al.: Bridging or Downstaging and LT for HCC

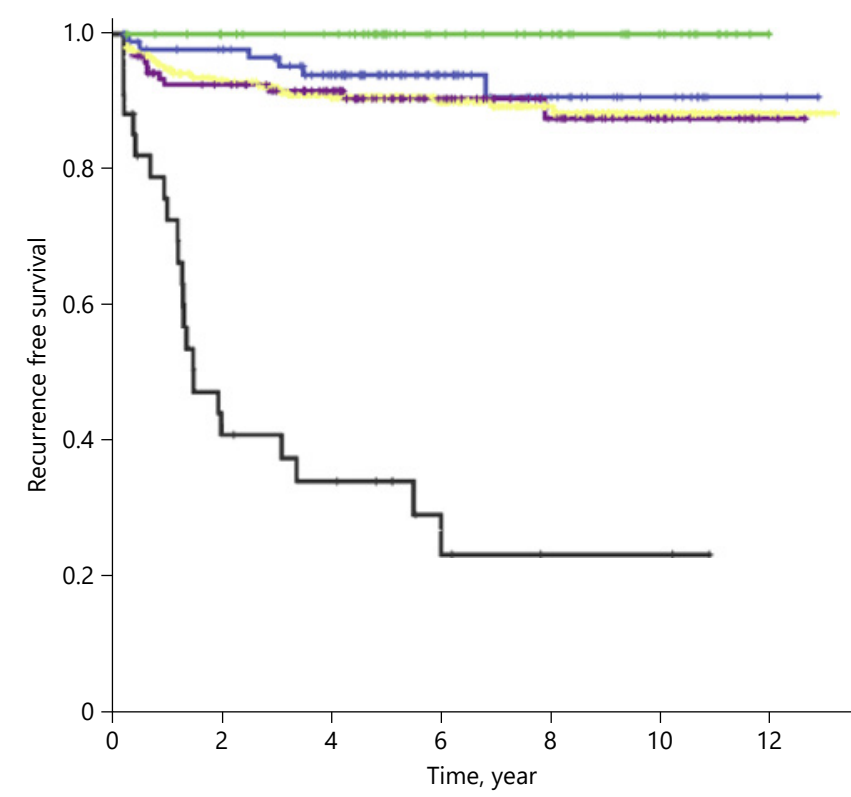

Recurrence free survival

_ Initial OPTN T1 HCC with bridging therapy $(n=91)$

_ Initial OPTN T1 HCC with primary LT $(n=54)$

1 year 3 years 5 years 10 years

Initial OPTN T2 HCC with bridging therapy $(n=350)$

$97.8 \% \quad 96.5 \% \quad 94.0 \% \quad 90.7 \%$

Initial OPTN T2 HCC with primary LT ( $n=123)$

a _ Initial OPTN T1 or T2 HCC with progression $(n=34)$

\section{$100 \%$}

$100 \%$

$100 \% \quad 100 \%$

$\begin{array}{llll}94.8 \% & 92.1 \% & 90.7 \% & 88.4 \%\end{array}$

$92.6 \% \quad 91.7 \%$

$90.5 \% \quad 87.6 \%$

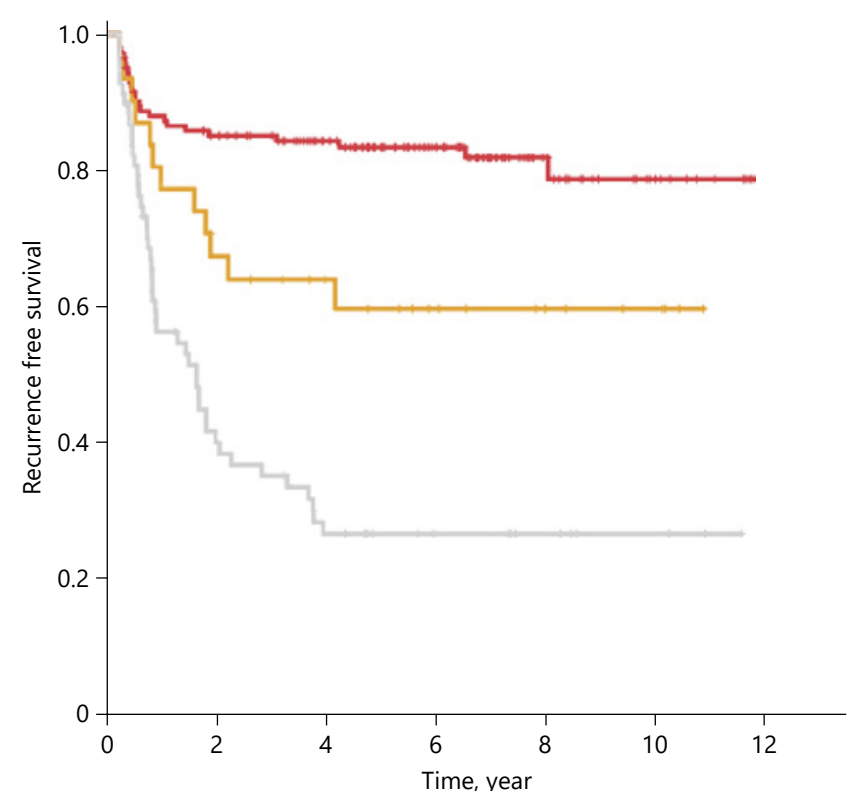

Recurrence free survival

1 year

— Initial OPTN T3 HCC with successful downstaging ( $n=144)$

$88.1 \%$

$77.4 \%$

$41.1 \%$

$34.2 \% \quad 23.5 \%$

$$
\text { b }
$$

_ Initial OPTN T3 HCC with primary LT $(n=31)$

$56.2 \%$

$\begin{array}{lll}3 \text { years } & 5 \text { years } & 10 \text { years } \\ 85.2 \% & 83.5 \% & 78.9 \% \\ 64.2 \% & 59.9 \% & 59.9 \% \\ 35.6 \% & 27.1 \% & 27.1 \%\end{array}$

Fig. 1. The RFS curves of patients who underwent LT with or without bridging or downstaging for HCC, stratified by initial OPTN stage and status after LRT: initial OPTN stage T1 or T2 (a) and initial OPTN stage T3 (b). RFS, recurrence-free survival; LT, liver transplantation; HCC, hepatocellular carcinoma; OPTN, Organ Procurement and Transplantation Network; LRT, locoregional therapy; SD, successful downstaging; DF, downstaging failure. 


\begin{tabular}{ll|l} 
Liver Cancer & \multicolumn{2}{l}{ Lancer 2020;9:721-733 } \\
\hline DOI: 10.1159/000507887 & $\begin{array}{l}\text { () 2020 The Author(s). Published by S. Karger AG, Basel } \\
\text { www.karger.com/lic }\end{array}$ \\
\hline
\end{tabular}

Lee et al.: Bridging or Downstaging and LT for HCC

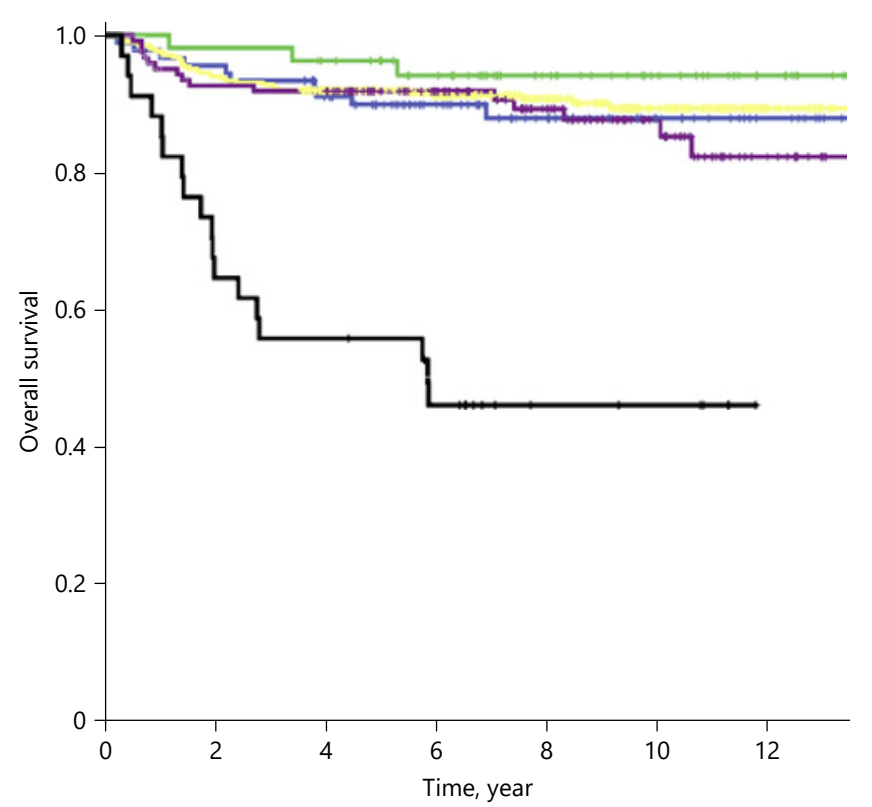

\section{Overall survival}

_ Initial OPTN T1 HCC with bridging therapy ( $n=91)$

_ Initial OPTN T1 HCC with primary LT $(n=54)$

Initial OPTN T2 HCC with bridging therapy $(n=350)$

_ Initial OPTN T2 HCC with primary LT $(n=123)$

a _ Initial OPTN T1 or T2 HCC with progression $(n=34)$

$\begin{array}{llll}1 \text { year } & 3 \text { years } & \text { 5 years } & 10 \text { years } \\ 97.8 \% & 93.4 \% & 89.9 \% & 87.9 \% \\ 98.1 \% & 96.3 \% & 94.2 \% & 94.2 \% \\ 97.7 \% & 92.9 \% & 92.0 \% & 89.4 \% \\ 95.1 \% & 91.9 \% & 91.9 \% & 87.7 \% \\ 92.8 \% & 68.1 \% & 50.2 \% & 47.9 \%\end{array}$

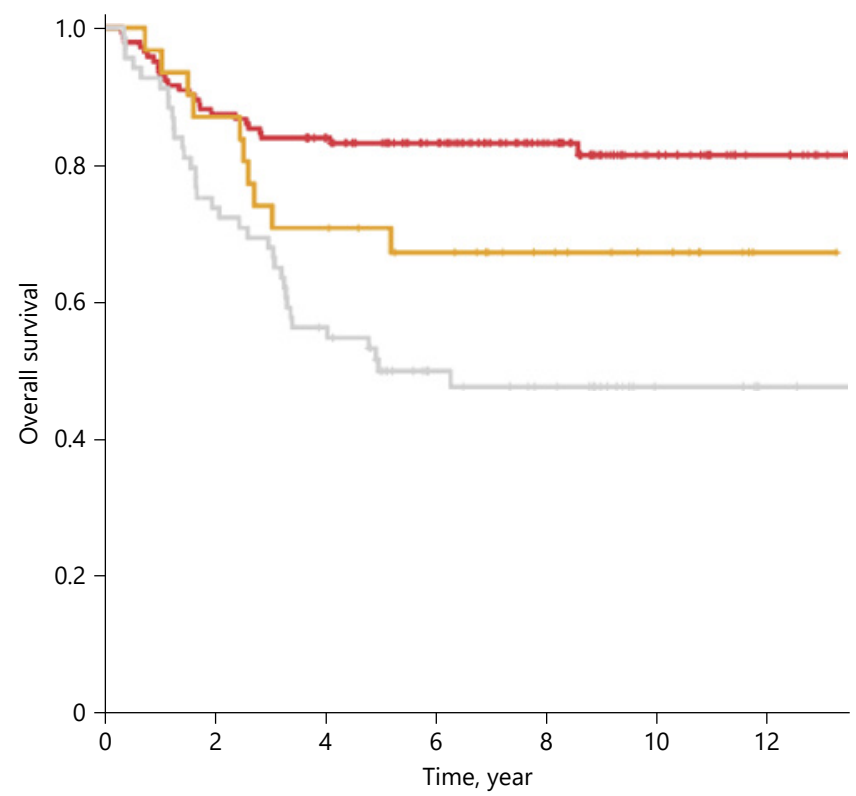

\section{Overall survival}

_ Initial OPTN T3 HCC with successful downstaging ( $n=144)$

$\begin{array}{llll}1 \text { year } & 3 \text { years } & \mathbf{5} \text { years } & 10 \text { years } \\ 94.4 \% & 84.0 \% & 83.3 \% & 81.6 \% \\ 96.8 \% & 74.2 \% & 71.0 \% & 67.4 \% \\ 95.2 \% & 73.0 \% & 55.0 \% & 52.5 \%\end{array}$

b_Initial OPTN T3 HCC with downstaging failure $(n=69)$

Fig. 2. The OS curves of patients who underwent LT with or without bridging or downstaging for HCC, stratified by initial OPTN stage and status after LRT: initial OPTN stage T1 or T2 (a) and initial OPTN stage T3 (b). OS, overall survival; LT, liver transplantation; HCC, hepatocellular carcinoma; OPTN, Organ Procurement and Transplantation Network; LRT, locoregional therapy; SD, successful downstaging; DF, downstaging failure. 
Table 2. Comparison of the characteristics between SD and DF or progression

\begin{tabular}{|c|c|c|c|}
\hline Patients & $\begin{array}{l}\text { SD } \\
(n=144)\end{array}$ & $\begin{array}{l}\text { DF or progression } \\
(n=103)\end{array}$ & $p$ value \\
\hline Age, years ${ }^{\mathrm{a}}$ & $54.3 \pm 7.0$ & $54.3 \pm 6.8$ & 0.976 \\
\hline Male & $120(83.3)$ & $91(88.3)$ & 0.361 \\
\hline \multicolumn{4}{|l|}{ Etiology of liver disease } \\
\hline Hepatitis B & $124(86.1)$ & $90(87.4)$ & \multirow[t]{4}{*}{0.941} \\
\hline Hepatitis C & $10(6.9)$ & $8(7.8)$ & \\
\hline Alcohol & $4(2.8)$ & $2(1.9)$ & \\
\hline Others & $6(4.2)$ & $3(2.9)$ & \\
\hline \multicolumn{4}{|l|}{ Child-Pugh classification } \\
\hline Class A & $80(55.5)$ & $35(34.0)$ & \multirow[t]{3}{*}{0.002} \\
\hline Class B & $40(27.8)$ & $47(45.6)$ & \\
\hline Class C & $24(16.7)$ & $21(20.4)$ & \\
\hline MELD score ${ }^{\mathrm{b}}$ & $9(7-14)$ & $11(8-16)$ & 0.013 \\
\hline Last AFP before LT, ng/mL & $3.9(10.1-46.0)$ & $49.9(9.5-554.0)$ & $<0.001$ \\
\hline \multicolumn{4}{|l|}{ Tumor characteristics on pretransplant imaging } \\
\hline Diameter of the largest viable tumor ${ }^{\mathrm{a}}$ & $1.1 \pm 1.2$ & $3.6 \pm 1.7$ & $<0.001$ \\
\hline Viable tumor, ${ }^{\mathrm{a}} n$ & $1.0 \pm 1.8$ & $5.3 \pm 5.7$ & \multirow[t]{2}{*}{$<0.001$} \\
\hline PET & $n=129$ & $n=94$ & \\
\hline Positivity & $50(38.8)$ & $46(48.9)$ & 0.135 \\
\hline \multicolumn{4}{|l|}{ LT after treatment } \\
\hline Types of treatment & & & \\
\hline TACE alone & $102(70.8)$ & $59(57.3)$ & \multirow[t]{5}{*}{0.004} \\
\hline RFA alone & $0(0)$ & $4(3.9)$ & \\
\hline Resection alone & $3(2.1)$ & $0(0)$ & \\
\hline Radiation alone & $1(0.7)$ & $0(0)$ & \\
\hline Combination & $38(26.4)$ & $40(38.8)$ & \\
\hline Radiologic complete response after treatment & $63(43.8)$ & $0(0)$ & \multirow[t]{2}{*}{$<0.001$} \\
\hline Tumor characteristics on pathology & $n=113$ & $n=103$ & \\
\hline \multicolumn{4}{|l|}{ Edmondson grade } \\
\hline I or II & $49(43.4)$ & $30(29.1)$ & \multirow[t]{2}{*}{0.034} \\
\hline III or IV & $64(56.6)$ & $73(70.9)$ & \\
\hline MVI & $14(12.4)$ & $43(41.7)$ & $<0.001$ \\
\hline Satellite nodule & $5(4.4)$ & $33(32.0)$ & $<0.001$ \\
\hline Interval from initial HCC diagnosis to LT, months $\mathrm{s}^{\mathrm{b}}$ & $12(4-26)$ & $19(10-47)$ & $<0.001$ \\
\hline
\end{tabular}

Unless otherwise indicated, data represent number of patients, with percentage in parentheses, and compared by using the $\chi^{2}$ test or Fisher's exact test. SD, successful downstaging; DF, downstaging failure; MELD, Model for End-Stage Liver Disease; AFP, alpha-fetoprotein; LT, liver transplantation; PET, positron emission tomography; TACE, transarterial chemoembolization; RFA, radiofrequency ablation; HCC, hepatocellular carcinoma; MVI, microvascular invasion. ${ }^{\mathrm{a}}$ Data are continuous variables, reported as means \pm standard deviations, and were compared by using the 2 -sample $t$ test. ${ }^{b}$ Data are continuous variables, reported as median with interquartile range, and were compared by using the Mann-Whitney $\mathrm{U}$ test.

with initial OPTN T3, the 5-year RFS rate was higher in patients with SD than in those with primary LT (83.5 vs. $59.9 \%, p=0.012$ ).

Transplanted patients with DF had a significantly lower 5-year RFS and OS rates after LT compared to those in patients with SD (27.1 vs. $83.5 \%, p<0.001 ; 55.0$ vs. $83.3 \%, p<0.001)$. Furthermore, the 5-year RFS and OS rates of patients with initial OPTN T1 or T2 with progression were significantly lower than those of patients with initial OPTN T3 with SD (34.2 vs. $83.5 \%, p<0.001 ; 50.2$ vs. $83.3 \%, p<0.001$ ). The characteristics of patients with SD and DF or progression are shown in Table 2. In the tumor characteristics on pathology, the DF or progression group had worse tumor grades and more frequent MVI and satellite nodule 


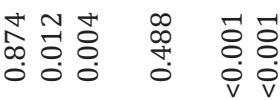

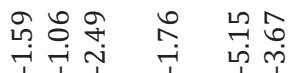

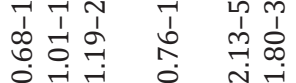

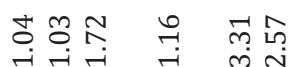

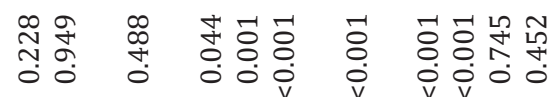

के

+น

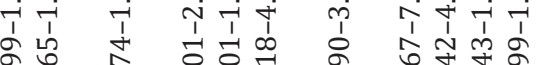

○ 0 O

芹

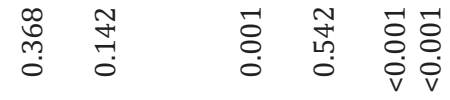

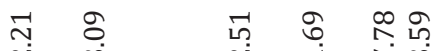

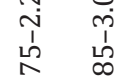

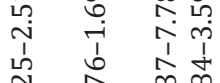

० 0 - 0 mi

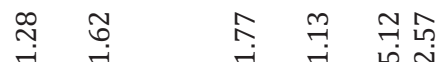

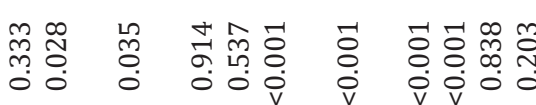

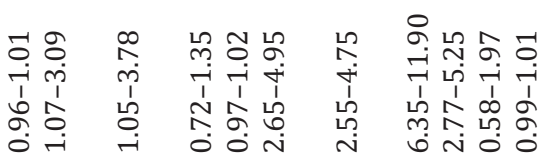

$\simeq \Xi \pm$

बून 
when compared with the SD group ( $p=0.034, p<0.001$, and $p<0.001$, respectively). In addition, the multivariate analysis using logistic regression demonstrated that age (odds ratio [OR]: $0.95,95 \%$ confidence interval [CI]: 0.93-0.97, $p<0.001$ ), last AFP before LT $<70$ ng/mL (OR: 2.18, 95\% CI: 1.06-4.46, $p=0.034$ ), absence of MVI (OR: 4.82, 95\% CI: $2.17-$ 10.73, $p<0.001$ ), and absence of satellite nodule (OR: 6.82, 95\% CI: 2.57-18.11, $p<0.001$ ) were significant independent predictors for SD (online suppl. Table 1).

\section{Pretransplant Predictors of RFS and OS}

In the multivariate analysis, last AFP before $\mathrm{LT} \geq 70 \mathrm{ng} / \mathrm{mL}$ (hazard ratio [HR]: $1.77,95 \%$ CI: $1.25-2.51, p=0.001$; HR: 1.72, 95\% CI: 1.19-2.49, $p=0.004$, respectively), pretransplant HCC status exceeding the Milan criteria (HR: 5.12, 95\% CI: 3.37-7.78, $p<0.001$; HR: 3.31, 95\% CI: 2.13-5.15, $p<0.001$, respectively), and PET positivity (HR: 2.57, 95\% CI: $1.84-3.59, p<$ 0.001; HR: $2.57,95 \%$ CI: $1.80-3.67, p<0.001$, respectively) were significant independent pretransplant predictors for worse outcomes in both RFS and OS (Table 3).

\section{Discussion}

Our study, which included a relatively large number of patients, indicates that SD was associated with improved RFS and OS in patients initially presenting with OPTN T3 HCC. Patients who experienced SD have pathologic features suggesting more favorable biology than those who experienced DF or progression. In the multivariate analysis, last AFP before $\mathrm{LT} \geq 70 \mathrm{ng} / \mathrm{mL}$, pretransplant HCC status exceeding the Milan criteria, and PET positivity were independent pretransplant predictors for worse RFS and OS.

In patients with initial OPTN T1, there has not yet been a study that directly compares LT with bridging versus primary LT in terms of long-term survival outcomes. Our comparative results demonstrated that the RFS and OS rates were not significantly different between LT with bridging therapy and primary LT, thus meeting the expectations recommended in an international consensus conference [3]. In a recent systemic review and meta-analysis of patients with initial OPTN T2 HCC [9], posttransplant HCC recurrence and OS were not significantly impacted by bridging versus proceeding directly to LT, which is consistent with our findings that the RFS and OS rates of patients who received LT with bridging therapy were similar to those who underwent primary LT.

Previous retrospective studies reported that the survival after LT in patients who had initial OPTN T3 HCC with subsequent SD was similar to that in patients who initially met the Milan criteria [16-20]. However, the definitions of SD and methods of radiologic response assessment had substantial heterogeneity among these studies. Previous prospective studies found that patients who experienced downstaging of tumors initially exceeding OPTN T2 but meeting their restriction criteria (Bologna or UCSF downstaging protocol) showed comparable outcomes to patients who met OPTN T2 HCC without downstaging [21, 22]. In our study, we defined SD as within the Milan criteria using mRECIST without a priori restriction of candidates for possible downstaging, and the RFS and OS rates of initial OPTN T3 HCC patients with SD were not significantly different compared to those of patients with initial OPTN stage T2 without bridging therapy, which is in line with a recent study by Chapman et al. [23].

There were limited data for comparison of survival outcomes of patients with OPTN T3 HCC with or without downstaging because receiving primary DDLT for patients with OPTN T3 HCC is rare. In cases of LDLT, LT for treating HCC in patients who exceeded the generally acceptable criteria (i.e., OPTN T3 with primary LT, initial OPTN T3 with DF, or initial OPTN $\mathrm{T} 1$ or T2 with progression) was performed upon the request of the patient and donor after a

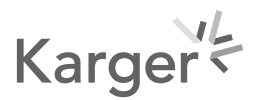


thorough explanation of the high recurrence probability. In our study consisting mostly of LDLT, OPTN T3 HCC patients with primary LT had a worse RFS than those with LT after SD. Furthermore, the long-term outcomes of LT in patients with DF or progression were disappointing, with a 5-year RFS and OS rates of approximately 30 and 50\%, respectively. Response to downstaging might be a surrogate of tumor biology and has been reported to be associated with outcomes after LT $[4,9,24,25]$. Our study confirmed that patients with SD had more favorable tumor biology (i.e., low histologic grade, absence of MVI, and satellite nodule) while patients with DF or progression presented more aggressive tumor histologic characteristics on explanted livers. Downstaging is not only a valid tool for morphological accepted criteria (within the Milan criteria) but also an additional selection tool for more favorable tumor biology and better prognosis.

Our study demonstrated that last AFP before LT $\geq 70 \mathrm{ng} / \mathrm{mL}$, pretransplant HCC status exceeding the Milan criteria, and PET positivity were independent prognostic factors for worse RFS and OS. Elevated AFP is a well-known prognostic marker associated with worse post-LT outcome and has been integrated into prognostic models after LT for HCC [26-28]. The Milan criteria remains the gold standard for determining the appropriate transplant strategy of HCC patients [29]. In patients treated with LRT prior to LT, the pretransplant Milan criteria included radiologic response to LRT in addition to morphological tumor size and number. In the previous studies, PET status has been shown to predict posttransplant tumor recurrence [30-32], which is similar to our study.

Our study has several limitations. First, it is a single-center retrospective study, in which selection bias is unavoidable. Second, most of the study population (87.7\%) had hepatitis B viral infection, and thus the results of our study may not be generalizable. Further studies in international multicenter settings that include a large number of patients with cirrhosis of various etiologies such as hepatitis $\mathrm{C}$ viral infection or alcohol consumption are needed. Third, we could not evaluate the dropout rate, wait time from listing to LT, and waitlist mortality in this study. Our center has adopted extended LDLT criteria for HCC because living donor grafts are considered dedicated gifts and are not subject to allocation systems as used for DDLT. Some patients with HCC underwent LDLT in spite of DF or progression after LRT. By including patients who underwent LT for HCC in excess of the generally acceptable criteria in our study, we could identify the worse outcomes of these patients. Accordingly, we could confirm that patients with OPTN T3 HCC should undergo LT after SD. Fourth, we did not compare the long-term outcomes based on therapeutic modalities used for bridging and downstaging. Lastly, we could not include PIVKA-II in the analyses because PIVKA-II was not routinely examined in all patients throughout the period of our study.

In conclusion, the impact of bridging therapy on survival outcomes is limited in patients with early-stage HCC, whereas OPTN T1 or T2 with progression provides worse prognosis. OPTN T3 should undergo LT after SD, and OPTN T3 with SD allows for acceptable long-term posttransplant outcomes.

\section{Statement of Ethics}

Each transplantation procedure was evaluated and approved by the local authorities and the Korean Network for Organ Sharing affiliated with the Ministry of Health and Welfare of the Republic of Korea, and this study was reviewed and approved by the institutional review board of our institution. The study protocol conforms to the ethical guidelines of the 1975 Declaration of Helsinki as reflected in a priori approval by the institution's human research committee. The requirement for informed consent was waived owing to the retrospective nature of the study.

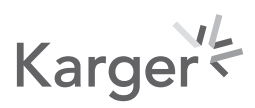




\section{Conflict of Interest Statement}

The authors have no conflicts of interest to declare.

\section{Funding Sources}

This research was supported by the Basic Science Research Program through the National Research Foundation (NRF) of Korea, funded by the Ministry of Science, ICT, and Future Planning (No. 2017R1E1A1A03070961).

\section{Author Contributions}

Gi-Won Song and Kyoung Won Kim had full access to all of the data in the study and take responsibility for the integrity of the data and the accuracy of the data analysis; Sunyoung Lee, Gi-Won Song, and Kyoung Won Kim were involved in the study concept and design, analysis and interpretation of data, and drafting of the manuscript; Sunyoung Lee, Gi-Won Song, Kyoung Won Kim, and Jae Hyun Kwon were involved in the acquisition of data; Shin Hwang, Ki-Hun Kim, Chul-Soo Ahn, Deok-Bog Moon, and Gil-Chun Park were involved in the critical revision of the manuscript for important intellectual content and material support; and Sung-Gyu Lee was involved in the study supervision.

\section{References}

1 Mazzaferro V, Regalia E, Doci R, Andreola S, Pulvirenti A, Bozzetti F, et al. Liver transplantation for the treatment of small hepatocellular carcinomas in patients with cirrhosis. N Engl J Med. 1996;334(11):693-9.

2 Lee SG, Moon DB. Living donor liver transplantation for hepatocellular carcinoma. Recent Results Cancer Res. 2013;190:165-79.

3 Clavien PA, Lesurtel M, Bossuyt PM, Gores GJ, Langer B, Perrier A. Recommendations for liver transplantation for hepatocellular carcinoma: an international consensus conference report. Lancet Oncol. 2012;13(1):e1122.

4 European Association for the Study of the Liver. EASL Clinical Practice Guidelines: management of hepatocellular carcinoma. J Hepatol. 2018;69:182-236.

5 Heimbach JK, Kulik LM, Finn RS, Sirlin CB, Abecassis MM, Roberts LR, et al. AASLD guidelines for the treatment of hepatocellular carcinoma. Hepatology. 2018;67(1):358-80.

6 Marrero JA, Kulik LM, Sirlin CB, Zhu AX, Finn RS, Abecassis MM, et al. Diagnosis, staging, and management of hepatocellular carcinoma: 2018 Practice Guidance by the American Association for the Study of Liver Diseases. Hepatology. 2018;68(2):723-50.

7 Pompili M, Francica G, Ponziani FR, Iezzi R, Avolio AW. Bridging and downstaging treatments for hepatocellular carcinoma in patients on the waiting list for liver transplantation. World J Gastroenterol. 2013;19(43): 7515-30.

8 Parikh ND, Waljee AK, Singal AG. Downstaging hepatocellular carcinoma: a systematic review and pooled analysis. Liver Transpl. 2015;21(9):1142-52.

9 Kulik L, Heimbach JK, Zaiem F, Almasri J, Prokop LJ, Wang Z, et al. Therapies for patients with hepatocellular carcinoma awaiting liver transplantation: a systematic review and meta-analysis. Hepatology. 2018;67(1): 381-400.

10 Bruix J, Sherman M. American Association for the Study of Liver Diseases: management of hepatocellular carcinoma: an update. Hepatology. 2011;53:1020-2.

11 European Association for the Study of the Liver, European Organisation for Research and treatment of Cancer. EASL-EORTC clinical practice guidelines: management of hepatocellular carcinoma. J Hepatol. 2012;56:90843.

12 Lencioni R, Llovet JM. Modified RECIST (mRECIST) assessment for hepatocellular carcinoma. Semin Liver Dis. 2010;30(1):52-60.

13 Bohm B, Voth M, Geoghegan J, Hellfritzsch H, Petrovich A, Scheele J, et al. Impact of positron emission tomography on strategy in liver resection for primary and secondary liver tumors. J Cancer Res Clin Oncol. 2004; 130:266-72.

14 Hwang S, Lee SG, Lee YJ, Sung KB, Park KM, Kim KH, et al. Lessons learned from 1,000 living donor liver transplantations in a single center: how to make living donations safe. Liver Transpl. 2006;12(6):920-7.

15 Hwang S, Moon DB, Ahn CS, Kim KH, Ha TY, Song GW, et al. Risk-based long-term screening for hepatocellular carcinoma recurrence after living donor liver transplantation. Transplant Proc. 2013;45(8):3076-84. 
Lee et al.: Bridging or Downstaging and LT for HCC

16 Otto G, Herber S, Heise M, Lohse AW, Mönch C, Bittinger F, et al. Response to transarterial chemoembolization as a biological selection criterion for liver transplantation in hepatocellular carcinoma. Liver Transpl. 2006; 12(8):1260-7.

17 Cillo U, Vitale A, Grigoletto F, Gringeri E, D'Amico F, Valmasoni M, et al. Intention-to-treat analysis of liver transplantation in selected, aggressively treated HCC patients exceeding the Milan criteria. Am J Transplant. 2007;7(4):972-81.

18 Chapman WC, Majella Doyle MB, Stuart JE, Vachharajani N, Crippin JS, Anderson CD, et al. Outcomes of neoadjuvant transarterial chemoembolization to downstage hepatocellular carcinoma before liver transplantation. Ann Surg. 2008;248(4):617-25.

19 De Luna W, Sze DY, Ahmed A, Ha BY, Ayoub W, Keeffe EB, et al. Transarterial chemoinfusion for hepatocellular carcinoma as downstaging therapy and a bridge toward liver transplantation. Am J Transplant. 2009;9(5): $1158-68$.

20 Lei J, Wang W, Yan L. Downstaging advanced hepatocellular carcinoma to the Milan criteria may provide a comparable outcome to conventional Milan criteria. J Gastrointest Surg. 2013;17(8):1440-6.

21 Ravaioli M, Grazi GL, Piscaglia F, Trevisani F, Cescon M, Ercolani G, et al. Liver transplantation for hepatocellular carcinoma: results of down-staging in patients initially outside the Milan selection criteria. Am J Transplant. 2008;8(12):2547-57.

22 Yao FY, Mehta N, Flemming J, Dodge J, Hameed B, Fix O, et al. Downstaging of hepatocellular cancer before liver transplant: long-term outcome compared to tumors within Milan criteria. Hepatology. 2015;61(6):1968-77.

23 Chapman WC, Garcia-Aroz S, Vachharajani N, Fowler K, Saad N, Lin Y, et al. Liver transplantation for advanced hepatocellular carcinoma after downstaging without up-front stage restrictions. J Am Coll Surg. 2017;224(4): 610-21.

24 Lo C. Downstaging of hepatocellular carcinoma before transplantation: an advance in therapy or just another selection criterion. Am J Transplant. 2008;8(12):2485-6.

25 Yao FY, Breitenstein S, Broelsch CE, Dufour JF, Sherman M. Does a patient qualify for liver transplantation after the down-staging of hepatocellular carcinoma? Liver Transpl. 2011;17(Suppl 2):S109-16.

26 Halazun KJ, Najjar M, Abdelmessih RM, Samstein B, Griesemer AD, Guarrera JV, et al. Recurrence after liver transplantation for hepatocellular carcinoma: a new MORAL to the story. Ann Surg. 2017;265(3):557-64.

27 Mehta N, Heimbach J, Harnois DM, Sapisochin G, Dodge JL, Lee D, et al. Validation of a risk estimation of tumor recurrence after transplant (RETREAT) score for hepatocellular carcinoma recurrence after liver transplant. JAMA Oncol. 2017;3(4):493-500.

28 Mazzaferro V, Sposito C, Zhou J, Pinna AD, De Carlis L, Fan J, et al. Metroticket 2.0 model for analysis of competing risks of death after liver transplantation for hepatocellular carcinoma. Gastroenterology. 2018; 154(1):128-39.

29 Mazzaferro V, Bhoori S, Sposito C, Bongini M, Langer M, Miceli R, et al. Milan criteria in liver transplantation for hepatocellular carcinoma: an evidence-based analysis of 15 years of experience. Liver Transpl. 2011; 17(Suppl 2):S44-57.

30 Yang SH, Suh KS, Lee HW, Cho EH, Cho JY, Cho YB, et al. The role of (18)F-FDG-PET imaging for the selection of liver transplantation candidates among hepatocellular carcinoma patients. Liver Transpl. 2006;12(11): 1655-60.

31 Kornberg A, Freesmeyer M, Bärthel E, Jandt K, Katenkamp K, Steenbeck J, et al. 18F-FDG-uptake of hepatocellular carcinoma on PET predicts microvascular tumor invasion in liver transplant patients. Am J Transplant. 2009;9(3):592-600.

32 Takada Y, Kaido T, Shirabe K, Nagano H, Egawa H, Sugawara Y, et al. Significance of preoperative fluorodeoxyglucose-positron emission tomography in prediction of tumor recurrence after liver transplantation for hepatocellular carcinoma patients: a Japanese multicenter study. J Hepatobiliary Pancreat Sci. 2017;24(1): 49-57. 